\title{
EVALUACIÓN DEL IMPACTO DE LA INTERVENCIÓN EDUCATIVA-MOTIVACIONAL "COMO JUGANDO" PARA PREVENIR LA OBESIDAD EN ESCOLARES DEL CERCADO DE LIMA: RESULTADOS AL PRIMER AÑO
}

\author{
Juan Pablo Aparco ${ }^{1,2, a}$, William Bautista-Olórtegui 1,a, Jenny Pillaca ${ }^{1, b}$
}

\begin{abstract}
RESUMEN
Objetivo. Determinar el impacto, al primer año, de la intervención educativa-motivacional "Como Jugando" sobre el índice de masa corporal (IMC), conocimientos en alimentación, niveles de actividad física y patrones de consumo alimentario en escolares del $1 .^{\circ}$ al $4 .^{\circ}$ grado de primaria, de cuatro instituciones educativas (IE) del Cercado de Lima. Materiales y métodos. Estudio cuasiexperimental, controlado. Se evaluó el IMC, conocimientos en nutrición, frecuencia de consumo de alimentos y nivel de actividad física. "Como Jugando" incluyó actividades lúdicas y vivenciales para escolares e involucró a docentes y padres. El impacto se estimó aplicando la prueba de McNemar para muestras relacionadas y modelos de regresión de diferencias en diferencias ajustados por variables relativas al escolar y la madre. Resultados. El estudio incluyó a 696 escolares de 2 IE de intervención y 2 IE de control, el puntaje Z de IMC y las prevalencias de obesidad no variaron durante el periodo de estudio. La intervención mostró impacto en mejorar los conocimientos en nutrición (OR: 1,46; IC95\%: 1,17-1,81) redujo el riesgo de tener un nivel malo de actividad física (OR: 0,63; IC95\%: 0,46-0,87) y mejoró los patrones de consumo de todos los alimentos, salvo verduras (grupo intervención). Conclusiones. La intervención "Como Jugando", al primer año de implementación, no redujo el Puntaje Z del IMC, sin embargo, tuvo impacto sobre los conocimientos en nutrición, redujo la probabilidad de tener un nivel malo de actividad física y mantuvo o aumentó la frecuencia adecuada del consumo de frutas, agua sin azúcar, jugos envasados y galletas dulces y saladas. Es necesario continuar evaluando la intervención "Como Jugando" para estimar el impacto sobre indicadores biológicos.
\end{abstract}

Palabras clave: Obesidad pediátrica; Educación primaria y secundaria; Intervención precoz (educación); Evaluación del impacto en la salud (fuente: DeCS BIREME).

\section{IMPACT EVALUATION OF EDUCATIONAL-MOTIVATIONAL INTERVENTION "COMO JUGANDO" TO PREVENT OBESITY IN SCHOOL CHILDREN OF CERCADO DE LIMA: RESULTS IN THE FIRST YEAR}

\begin{abstract}
Objective. To determine the impact of the educational-motivational intervention "Como jugando" on the body mass index (BMI), knowledge on nutrition, physical activity levels, and eating patterns in students from the first to fourth grades of four educational institutions (Els) of Cercado de Lima after the first year of the intervention. Materials and methods. This was a quasi-experimental, controlled study. BMI, knowledge on nutrition, frequency of food consumption, and level of physical activity were evaluated. The "Como jugando" program included playful and experiential activities for school children and involved both teachers and parents. The impact was estimated by applying the McNemar test for related samples and regression models for the analysis of differences in adjusted variables related to children and their mothers. Results. The study included 696 school children from two experimental Els and two control Els. The Z-score for BMI and the prevalence of obesity did not vary during the study period. The intervention improved the knowledge of nutrition [odds ratio (OR): $1.46 ; 95 \%$ confidence interval (Cl): 1.17-1.81], reduced the likelihood of poor levels of physical activity (OR: $0.63 ; 95 \% \mathrm{Cl}: 0.46-0.87$ ), and improved the consumption patterns for all food categories except vegetables in the intervention group. Conclusions. The "Como jugando" intervention, in the first year of implementation, did not reduce the Z-score for BMI but improved the knowledge of nutrition, reduced the probability of poor levels of physical activity, and maintained or increased the consumption of fruits, sugar-free water, packaged juices, and sweet and savory cookies. Therefore, the "Como jugando" intervention should be better studied in order to estimate its impact on biological indicators.
\end{abstract}

Key words: Pediatric obesity; Education; Primary and secondary; Early intervention (education); Health impact assessment (source: MeSH NLM).

\footnotetext{
Centro Nacional de Alimentación y Nutrición, Instituto Nacional de Salud. Lima, Perú.
}

Departamento Académico de Nutrición, Facultad de Medicina, Universidad Nacional Mayor de San Marcos. Lima, Perú.

Licenciado en Nutrición, ${ }^{\mathrm{b}}$ estadística.

Recibido: 01/11/2016 Aprobado: 06/09/2017 En línea: 29/09/2017

Citar como: Aparco JP, Bautista-Olórtegui W, Pillaca J. Evaluación del impacto de la intervención educativa-motivacional “Como jugando” para prevenir la obesidad en escolares del Cercado de Lima: resultados al primer año. Rev Peru Med Exp Salud Publica. 2017;34(3):386-94. doi: 10.17843/rpmesp.2017.343.2472 


\section{INTRODUCCIÓN}

La obesidad es, actualmente, uno de los mayores problemas de salud pública en el mundo ${ }^{(1)}$, ya que su gravedad y duración desde la infancia son predictores importantes de enfermedades cardiovasculares, diabetes mellitus 2 , cáncer, entre otras enfermedades no transmisibles ${ }^{(2)}$.

La prevalencia de obesidad infantil mundial, entre 1980 y 2013 , aumentó cerca de $50 \%{ }^{(3)}$; esta tendencia se refleja en nuestro país donde la obesidad en niños de 5 a 9 años, entre 2007 y 2014 , aumentó en $92 \%{ }^{(4)}$. El incremento de la obesidad obedece a múltiples factores, sin embargo, el entorno obesogénico se reconoce como el agente etiológico más importante ya que aumenta la disponibilidad de calorías y condiciona un mayor sedentarismo ${ }^{(5)}$.

El tratamiento de la obesidad es complejo y tiene baja efectividad tanto en niños como en adultos, por lo que es necesario desarrollar programas preventivos que resulten más costo-efectivos ${ }^{(6)}$. La escuela es un escenario adecuado para realizar intervenciones contra la obesidad, porque es donde los escolares pasan gran parte de su tiempo, toman algunas de las comidas y son educados de manera natural sobre estilos de vida saludables; además, junto al ámbito familiar son los espacios educativos que más influyen en la adquisición de hábitos alimentarios y estilos de vida saludable ${ }^{(7,2)}$.

A pesar de que se han desarrollado múltiples proyectos basados en la escuela para prevenir la obesidad, la evidencia actual muestra que la mayoría no tiene efecto para reducir el índice de masa corporal (IMC); entre las razones que explican el insuficiente efecto de las intervenciones, se encuentran que fueron de corto plazo, con un enfoque aislado (alimentación o actividad física), con objetivos simultáneos de prevención y tratamiento de obesidad y/o modelos educativos que abordaban el problema solo como falta de conocimiento ${ }^{(8,9)}$.

En Latinoamérica se han implementado intervenciones integrales para prevenir la obesidad infantil con resultados diversos; en Chile, Kain et al. (10) y Ratner et al. (11) encontraron reducciones significativas del puntaje $Z$ de IMC en los escolares intervenidos, mientras que Vio et al. (122 reportaron reducción en el puntaje $Z$ de IMC en algunos subgrupos, siendo más relevante el efecto en la mejora de conocimientos en nutrición y reducción del consumo de alimentos no saludables. En México, Shama-Levy et al. (13) mostraron la efectividad de una estrategia que promueve actividad física y alimentación saludable para mantener el IMC en el grupo de intervención; mientras que AlvirdeGarcia et al., en un ensayo comunitario de 3 años para promover estilos de vida saludable ${ }^{(14)}$, encontraron un menor incremento del IMC en el grupo de intervención al final de periodo; en tanto Safdie et al., en otro ensayo comunitario de 18 meses en escolares, no encontraron

\section{MENSAJES CLAVE}

Motivación para realizar el estudio. A pesar de que en el Perú la obesidad en escolares tiene una tendencia creciente, existe limitada información de que intervenciones funcionan, por lo que urge generar evidencia sobre modelos efectivos contra la obesidad en el contexto de nuestro país.

Principales hallazgos. "Como Jugando" tuvo impacto sobre los conocimientos en nutrición, protegió de un nivel malo de actividad física, mejoró el consumo de frutas y agua sin azúcar y mantuvo un consumo bajo de jugos envasados y galletas dulces y saladas.

Implicancias. Intervenciones que incluyan actividades lúdicas y vivenciales para el aprendizaje significativo en los niños e involucren a padres de familia y docentes podrían ser efectivas para prevenir la obesidad.

efectos sobre el IMC, aunque la ingesta de alimentos no saludables disminuyó significativamente ${ }^{(15)}$.

Con estos antecedentes, se desarrolló la intervención educativa-motivacional "Como Jugando" con un horizonte de tiempo de tres años, un enfoque integral de alimentación y actividad física y fundamentada en la teoría de aprendizaje social, que aborda los mediadores de la conducta ${ }^{(16)}$ y el constructivismo, para lograr un aprendizaje significativo en los niños, vinculando sus saberes previos con los contenidos de la intervención y generando un entorno favorable al aprendizaje a través de experiencias lúdicas y vivenciales vinculadas al entorno de los escolares ${ }^{(17,18)}$ para motivar la elección de alimentos saludables y actividades no sedentarias. Varios estudios que aplican la teoría de aprendizaje social en escolares muestran efectividad de este modelo educativo ${ }^{(9,13,15)}$.

En nuestro país, a pesar de que la obesidad muestra una tendencia creciente, la evidencia disponible sobre evaluación de intervenciones para prevenir la obesidad en escolares aún es insuficiente. Por ello, el objetivo del estudio fue evaluar el impacto, al primer año, de la intervención "Como Jugando" para reducir el IMC, incrementar los conocimientos en alimentación y mejorar los patrones de consumo alimentario y los niveles de actividad física en escolares del $1 .^{\circ}$ al $4 .^{\circ}$ grado de primaria de instituciones públicas del Cercado de Lima.

\section{MATERIALES Y MÉTODOS}

\section{DISEÑO DEL ESTUDIO}

Estudio cuasiexperimental, de diseño prepruebaposprueba y grupos intactos ${ }^{(19)}$ en cuatro instituciones educativas públicas (IE) mixtas de nivel primario del Cercado de Lima. El primer año de intervención se asignaron dos IE a intervención y dos a control; el 
segundo año tres se asignaron a intervención y uno a control y el tercer año todas las IE recibieron la intervención. El presente estudio muestra los resultados del primer año. La evaluación basal se realizó en marzo de 2014, la intervención se desarrolló entre abril y noviembre de 2014 y la evaluación final en diciembre del mismo año.

\section{POBLACIÓN DE ESTUDIO}

Escolares de $1 .^{\circ}$ a $4 .^{\circ}$ grado de 4 IE mixtas y de nivel primario del Cercado de Lima.

\section{MUESTRA}

Se estimó el tamaño de muestra considerando detectar una reducción de 0,3 desviaciones estándar (DE) en el promedio del puntaje $Z$ del IMC de los escolares del grupo de intervención ${ }^{(11)}$. Se aplicó una tasa de rechazo del $25 \%$, considerando posibles pérdidas y la varianza del indicador IMC $\left(s^{2}=1,36\right)$ calculado a partir de los datos de niños de 6 a 10 años de la encuesta ENAHO $2010^{(20)}$. Se estimó que cada grupo estaría formado por 316 niños.

\section{RECLUTAMIENTO DE ESCUELAS}

Se consideraron IE de nivel primario que: a) estuvieran ubicadas en el Cercado de Lima; b) fueran mixtas; c) tengan más de 100 alumnos por turno de clases; d) tengan dos o más aulas por grado, y d) no sean beneficiarias del Programa Qali Warma, ya que el programa provee desayuno a los escolares lo cual podría confundir los resultados del estudio.

De un listado inicial de 16 IE ubicadas en el Cercado de Lima, cuatro cumplían con los criterios de inclusión; dos se asignaron a intervención y dos a control. La intervención no se asignó aleatoriamente, las dos IE con intervención fueron las que se comprometieron a: a) dar facilidades (ambientes y tiempo) para desarrollar la intervención; b) modificar el entorno alimentario y de actividad física de la escuela; c) apoyar la capacitación en alimentación y actividad física a docentes y padres de familia y d) permitir la evaluación de los escolares y las actividades de la intervención.

\section{RECLUTAMIENTO DE ESCOLARES}

Las actividades de "Como Jugando" se desarrollaron en todas las aulas de $1 .^{\circ}$ a $4 .^{\circ}$ grado de primaria de las $2 \mathrm{IE}$ asignadas a intervención. Para la evaluación basal y de resultados se incluyeron a todos los escolares $1 .^{\circ}$ a $4 .^{\circ}$ grado de primaria, sin problemas posturales o ausencia de miembros inferiores que impidieran tomar los datos antropométricos, y con consentimiento informado de los padres para participar en el estudio. La tasa de rechazo fue inferior al $5 \%$.

\section{INTERVENCIÓN}

\section{EL PROYECTO “COMO JUGANDO”:}

El objetivo del proyecto "Como Jugando" era brindar conocimientos y habilidades, a escolares de $1 .^{\circ}$ a $4 .^{\circ}$ grado de primaria, para elegir adecuadamente alimentos saludables y realizar actividad física moderada; para ello, se desarrollaron los siguientes componentes:

a. Componente alimentario. Difusión de la norma de alimentos saludables en el quiosco escolar a los vendedores de alimentos y bebidas dentro de la IE, así como a docentes y padres de familia. Seguimiento de la implementación de la norma en la IE.

b. Componente de actividad física. Capacitación a docentes de educación física para desarrollar las clases con actividades motivadoras e inclusivas, que permitan participar a todos los alumnos de la activación física. Identificar "oportunidades" para desarrollar actividad física y dotar con implementos deportivos a los espacios para desarrollar la activación física.

c. Componente de información educación y comunicación en nutrición. Desarrollo de talleres lúdicos y vivenciales dirigido a los escolares, con el objetivo de formar capacidades para elegir alimentos saludables y realizar actividad física. Capacitación a docentes y padres de familia sobre los ejes temáticos de alimentación, actividad física y su participación en actividades de la intervención "Como Jugando".

d. Componente de motivación. Enfoque motivador para el desarrollo de temas de alimentación, nutrición y actividad física a través de cuentos y teatro de títeres basado en la teoría de aprendizaje entre pares ${ }^{(16)}$. Para implementar esta estrategia se desarrollaron los personajes de la intervención: Kike Pike, Kinita y Golosito, así como los guiones e historias que presentaban situaciones problemáticas cotidianas, las cuales permitían al escolar participar activamente en la solución para ayudar al personaje del cuento e interiorizar los mensajes clave. Para los docentes se desarrollaron talleres que les permitieron reforzar sus conocimientos en nutrición.

Estos componentes se elaboraron considerando las necesidades percibidas de los escolares y docentes, y los resultados del estudio basal (4) que permitieron priorizar las conductas factibles de modificar y los mensajes clave para escolares, docentes y padres de familia.

Los materiales educativos desarrollados para el proyecto "Como Jugando" incluyen: guía educativa para el docente, guía de actividades vivenciales para padres, ficha de seguimiento de las actividades vivenciales, fichas de trabajo para los alumnos, cinco guiones de teatro de títeres, el nutricontador del consumo de frutas y verduras para registrar el consumo semanal de estos 
alimentos y el activómetro, un material educativo para mostrar a los niños los beneficios de realizar actividades no sedentarias.

\section{DESCRIPCIÓN DE LA INTERVENCIÓN:}

Las actividades de "Como Jugando" fueron desarrolladas por un equipo de trabajo compuesto por una educadora y dos nutricionistas. La intervención se realizó en el horario escolar regular y constaba de:

a. Sesiones educativas de alimentación saludable y actividad física. Se desarrollaron cinco sesiones educativas (tres de alimentación saludable y dos de actividad física). La estructura de la sesión incluía la etapa inicial, en la cual se usaban materiales educativos (maquetas de alimentos, implementos deportivos y lúdicos) para el recojo de saberes previos; luego, el desarrollo de los contenidos (a través del teatro de títeres con guiones basados en un diseño pedagógico que abordaba las competencias de cada sesión) y el cierre (en donde se desarrollaba la evaluación del aprendizaje y la actividad vivencial). Todo el proceso duraba 50 min. La sesión educativa se desarrolló por cada grado y sección en cada una de las aulas, con una periodicidad mensual (excepción de julio y agosto por vacaciones escolares). Los alumnos presentes eran como máximo 30 por cada sección. En total, se desarrollaron 125 sesiones educativas durante el año 2014.

b. Capacitación a docentes. Se capacitó a los docentes de cada IE sobre contenidos de alimentación saludable, cómo reforzar los contenidos de la intervención y en la aplicación de las fichas de trabajo vivenciales para desarrollar con los padres. Adicionalmente, se capacitó a los profesores de educación física en sesiones inclusivas de actividad física, y se les proporcionó un kit a actividad física (conos, pelotas, redes, hula hula, soguillas, redecillas) y se ubicaron espacios en los que se pintaron, mundos, aviones y escaleras, o se entregaron secuencias de juegos en gigantografías para realizar actividad física durante el recreo.

c. Capacitación a través de escuela de padres. Se desarrollaron contenidos en alimentación saludable y actividad física dirigida a padres de familia; sin embargo, dado que en las dos IE de intervención, la asistencia de los padres alcanzaba un $30 \%$, el equipo decidió realizar visitas a los domicilios y centros de trabajo de cada padre de familia con la finalidad de brindarle información sobre la intervención, explicarle el rol que debía cumplir en las actividades a desarrollar con su hijo, cómo usar la guía de actividades vivenciales para padres y darle orientación nutricional.

d. Recreosactivos. Dos vecesala semanaun nutricionista y una educadora visitaban las IE de intervención para desarrollar durante el recreo una sesión activación física, para ello, se ponía a disposición de los alumnos el kit de actividad física y se organizaban rutinas y concursos en los mundos, aviones y escaleras pintados en los patios. Inicialmente, la animación sociocultural la desarrollaba el equipo del proyecto, sin embargo, con el tiempo, los agentes escolares de alimentación saludable se encargaban de desarrollar los recreos activos.

e. Formación de agentes escolares de alimentación saludable. Se desarrolló la estrategia de formación de agentes-niños que apoyaban el desarrollo de las sesiones educativas, realizaban teatro de títeres y la animación sociocultural de los recreos activos.

\section{VARIABLES}

Las variables consideradas para determinar el impacto de la intervención se describieron con detalle en una publicación anterior ${ }^{(4)}$ aquí se resumen algunas características:

Resultados principales: composición corporal.

a. IMC. Se midieron el peso y la talla de todos los escolares de $1 .^{\circ}$ a $4 .^{\circ}$ de primaria. Se usaron balanzas electrónicas (SECA®) con una resolución de $0,1 \mathrm{~kg}$ y tallímetros modelo OMS con una resolución de $0,1 \mathrm{~cm}$, siguiendo las pautas del Instituto Nacional de Salud (INS). El estado nutricional se obtuvo con base al puntaje Z del IMC para edad de cada niño y se clasificó según lo recomendado por OMS: $\leq 3 \mathrm{DE}$, delgadez extrema; $\leq 2 \mathrm{DE}$, bajo peso; entre -2 y $1 \mathrm{DE}$, normal; $>1$ $\mathrm{DE}$, sobrepeso y $>2 \mathrm{DE}$, obesidad.

b. Índice cintura/talla. Se midió el perímetro abdominal ubicando la distancia media entre el borde inferior de la última costilla y el borde superior de la cresta ilíaca. Se usó una cinta métrica de fibra de vidrio con una longitud de $200 \mathrm{~cm}$ y una resolución de $1 \mathrm{~mm}$. Los niveles de riesgo del índice cintura/talla se clasificaron con los puntos de corte propuestos por Muñoz-Cano ${ }^{(21)}$.

Resultados secundarios: conductas en alimentación y actividad física.

a. Patrones de consumo de alimentos. Este patrón fue aplicado por encuestadores entrenados en la metodología de frecuencia de consumo de alimentos cualitativo. Se entrevistó al niño y la madre. El cuestionario fue estructurado en torno a una lista cerrada de alimentos, bocaditos y bebidas clasificados en siete grupos: a) alimentos con dos grupos: frutas frescas y verduras; b) bocaditos con dos grupos: galletas dulces y galletas saladas, y c) bebidas con tres grupos: jugos/bebidas envasadas, gaseosas y agua sin azúcar. La frecuencia de consumo se consideró adecuada: a) para los grupos de frutas, verduras y agua sin azúcar: cuando el consumo fue de cinco o más veces a la semana; b) demás grupos: cuando el consumo fue de menos de dos veces a la semana. Ambos criterios se basaron en las recomendaciones 
de la Sociedad Española de Nutrición Comunitaria para niños y adolescentes ${ }^{(22)}$.

b. Conocimientos en nutrición. Encuestadores entrenados aplicaron un cuestionario de conocimientos en nutrición en las dos evaluaciones del 2014. El cuestionario fue validado por expertos y adecuado en campo para su fácil comprensión, consistió en cuatro preguntas para niños de $1 .^{\circ}$ y $2 .^{\circ}$ grado y ocho preguntas para niños de $3 .^{\circ}$ y $4 .^{\circ}$ grado, a través de diseños gráficos con imágenes de alimentos para facilitar la comprensión. Los temas incluidos fueron alimentación saludable, grupos de alimentos, lonchera escolar y las comidas del día. Se evaluó la capacidad para identificar y seleccionar los alimentos y la identificación de conductas que favorecían el cuidado de su salud. El conocimiento se definió como adecuado si respondían adecuadamente el $75 \%$ de las preguntas, e inadecuado si los aciertos fueron menores.

c. Niveles de actividad física. Se determinaron durante los días de clases y se aplicó el cuestionario de actividad física del Instituto de Nutrición y Tecnología de los Alimentos de Chile ${ }^{(23)}$. La calificación se realizó según puntajes aplicados en la evaluación basal (4) considerando nivel bueno: de 7 a 10 puntos, regular: de 4 a 6 puntos y malo: menor o igual a 3 puntos

\section{ANÁLISIS ESTADÍSTICO}

El estudio descriptivo se realizó en función de frecuencias y porcentajes (variables cualitativas) y medidas de tendencia central (media $[\bar{X}] \pm D E$ ) para las variables cuantitativas. Se usó la prueba de Shapiro-Wilk para evaluar la normalidad de la distribución de las variables principales. En el caso de las variables de consumo de alimentos se comparó su evolución en el tiempo aplicando la prueba de McNemar (muestras pareadas). Para determinar el impacto de la intervención se realizó un análisis de dobles diferencias (la primera diferencia entre etapas y la segunda diferencia entre grupos) para estimar el efecto atribuible de la intervención sobre los indicadores principales ${ }^{(24)}$ puntaje $z$ de IMC e índice de cintura/talla, asimismo para los indicadores secundarios conocimientos en nutrición y nivel de actividad física se utilizaron modelos logísticos binomiales para datos panel utilizando el comando "xtlogit". La estimación se ajustó por pobreza en el hogar ( $\geq 1$ necesidad básica insatisfecha [NBI]), edad y sexo del escolar, edad, años de estudio y situación laboral de la madre. En todos los casos se consideró los intervalos de confianza al 95\% (IC 95\%) y el valor $p<0.05$ como nivel de significancia estadística. El análisis estadístico se realizó mediante el programa estadístico STATAMMP 14.0.

\section{ASPECTOS ÉTICOS}

El protocolo de estudio fue revisadoy aprobado por el Comité Institucional de Ética en Investigación del INS (expediente $\mathrm{N}$ Ol-044-13). Se coordinó con la dirección de cada IE para el desarrollo de trabajo de campo e implementación de la intervención. Para la recolección de datos y toma de muestras, se solicitó previamente el consentimiento informado a ambos padres de los escolares.

\section{RESULTADOS}

En la figura 1 se observa el flujo de la muestra desde el reclutamiento de IE hasta la evaluación de resultados en diciembre de 2014. Se aprecia que se enrolaron 824

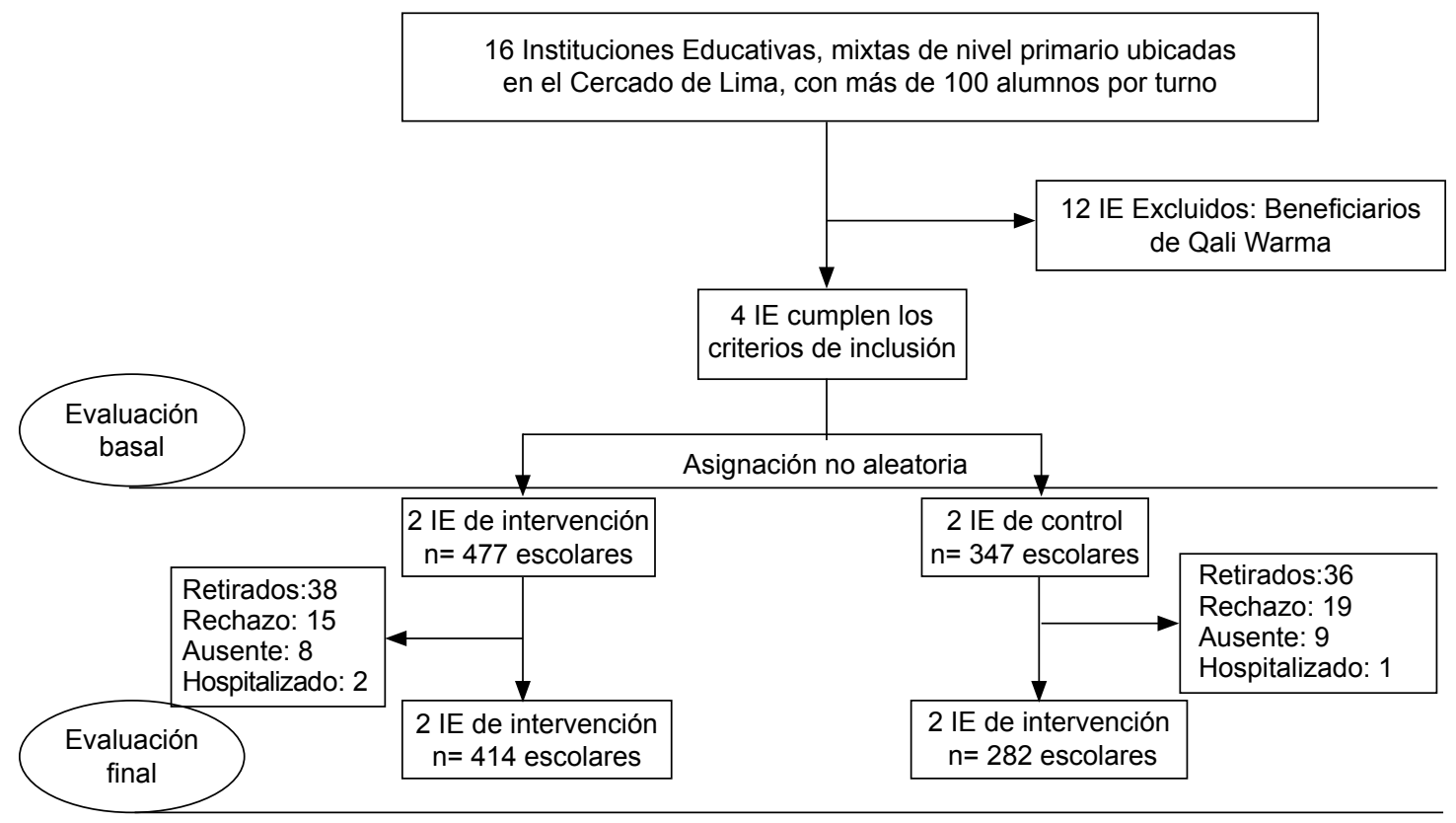

Figura 1. Flujograma de la muestra 
escolares en la evaluación inicial (477 en intervención y 347 en control) y que 696 escolares (414 en intervención y 282 en control) completaron las dos evaluaciones de marzo y noviembre de 2014.

Respecto a las características basales de los escolares y madres de los dos grupos de estudio (Tabla 1), se encontraron diferencias significativas en las variables IMC para edad, perímetro abdominal y proporción de madres que trabajan.

En el caso de la implementación de la intervención, el 91,6\% (488/533) de los escolares recibió las cinco sesiones educativas en las 2 IE de intervención, mientras que el $83,9 \%(447 / 533)$ de padres recibió orientación sobre los contenidos de la intervención.

Al evaluar los efectos de la intervención motivacional sobre la frecuencia de consumo de alimentos, la tabla 2 muestra que el consumo adecuado de frutas se mantuvo igual en el grupo de intervención, mientras que en el control esta conducta se redujo significativamente (-10 puntos porcentuales [pp]); en el caso de verduras, el comportamiento fue opuesto, pues se redujo significativamente en el grupo de intervención (-10 pp). Respecto al consumo adecuado de agua sin azúcar, también se aprecian efectos importantes, así, en el grupo de intervención hubo un aumento significativo (6 pp), mientras que en el grupo de control se redujo en 10 pp.

Por su parte, la conducta recomendada de consumir galletas dulces y saladas (menos de 2 días en la semana) se mantuvo en el grupo de intervención, en tanto que en el grupo de control se redujo significativamente (-11 pp y -12 pp respectivamente). En cuanto a consumo "adecuado" de los jugos envasados, no hubo variaciones en el grupo de intervención y sí se redujo significativamente en el grupo de control (10 pp).

Respecto a la prevalencia de obesidad, se encontró que en las IE de intervención la obesidad aumentó de 27,1 a $29 \%$ (1,9 pp), mientras que en las IE de control aumentó de 22,7 a $24,5 \%$ (1,8 pp); en tanto, el sobrepeso se redujo en las IE de intervención de 28 a $25,1 \%$ (-2,9 pp) y en las IE de control se redujo de 18,8 a 18,1 (-0,7 pp). Ningún cambio resultó significativo.

En el análisis multivariado (Tabla 3) para conocer el impacto de "Como Jugando" sobre el conocimiento en nutrición y actividad física, el modelo incluyó como variables de ajuste: pobreza del hogar, edad y sexo del niño; edad, años de estudio y condición laboral de la madre. Se observó que, tanto en el modelo crudo como ajustado, la intervención aumenta en 1,4 veces más la probabilidad de tener conocimiento adecuado en nutrición; asimismo, reduce en $37 \%$ la probabilidad de tener un nivel de actividad física mala.

Por su parte, la intervención educativa-motivacional no tuvo impacto sobre los indicadores principales como puntaje Z de IMC o el índice de cintura/talla, este resultado se mantuvo aún en el modelo ajustado (Tabla 4).

\section{DISCUSIÓN}

El estudio aporta evidencia sobre el impacto de la intervención educativa-motivacional "Como Jugando" sobre indicadores nutricionales de escolares de nivel primario.

Tabla 1. Características basales de los escolares, por grupo de estudio

\begin{tabular}{|c|c|c|c|}
\hline Características & $\begin{array}{c}\text { Intervención } \\
\bar{x} \text { (IC 95\%) } \\
\end{array}$ & $\begin{array}{c}\text { Control } \\
\bar{x}(\text { IC 95\%) } \\
\end{array}$ & Valor de $\mathbf{p}^{\dagger}$ \\
\hline Escolares & $n=414$ & $n=282$ & \\
\hline Edad (años) & $7,4(7,3-7,5)$ & $7,6(7,4-7,5)$ & 0,113 \\
\hline Peso (kg) & $29,3(28,4-30,0)$ & $28,4(27,4-29,3)$ & 0,117 \\
\hline Talla (cm) & $124,4(123,5-125,2)$ & $124,5(123,4-125,4)$ & 0,867 \\
\hline IMC/Edad (score Z) & $1,19(1,06-1,3)$ & $0,88(0,7-1,04)$ & $0,002^{*}$ \\
\hline Perímetro abdominal (cm) & $63,6(62,8-64,4)$ & $61,8(60,7-62,7)$ & $0,002^{*}$ \\
\hline Hemoglobina (g/dL) & $12,5(12,4-12,6)$ & $12,5(12,4-12,6)$ & 0,921 \\
\hline Escolares mujeres & $182 / 414(43,9 \%)$ & $122 / 282(43,3 \%)$ & 0,855 \\
\hline \multicolumn{4}{|l|}{ Madres de escolares $\ddagger$} \\
\hline Edad de la madre (años) & $36,3(35,5-37,1)$ & $36,1(35,0-37,1)$ & 0,711 \\
\hline Años de estudio de la madre & $10,1(9,7-10,3)$ & $10,3(10,0-10,6)$ & 0,991 \\
\hline Madre trabaja & $294 / 414(71,0 \%)$ & $174 / 282(61,7 \%)$ & $0,011^{*}$ \\
\hline Pobreza en el hogar ( $\geq 1 \mathrm{NBI}$ ) & $117 / 414(28,3 \%)$ & $81 / 282(28,7 \%)$ & 0,894 \\
\hline
\end{tabular}

* $p<0,05$

† Prueba t/Mann-Whitney

‡ El número de madres es igual al número de niños

$\overline{\mathrm{x}}$ : media aritmética; IC 95\%: intervalo de confianza al 95\%

NBI: Necesidad básica insatisfecha 
Tabla 2. Evolución de los patrones de consumo en escolares de IE de intervención y de control

\begin{tabular}{|c|c|c|c|c|c|c|c|c|}
\hline \multirow[b]{2}{*}{ Indicador } & \multicolumn{3}{|c|}{ Intervención } & \multirow[b]{2}{*}{ p valort } & \multicolumn{3}{|c|}{ Control } & \multirow[b]{2}{*}{ p valort } \\
\hline & $\begin{array}{c}\text { Antes } \\
(\%)\end{array}$ & $\begin{array}{c}\text { Después } \\
(\%)\end{array}$ & $\begin{array}{c}\text { Cambio } \\
(\%)\end{array}$ & & $\begin{array}{c}\text { Antes } \\
(\%)\end{array}$ & $\begin{array}{c}\text { Después } \\
(\%)\end{array}$ & $\begin{array}{c}\text { Cambio } \\
(\%)\end{array}$ & \\
\hline Consumo de frutas $\geq 5$ días/semana & 56,5 & 58,5 & 2,0 & 0,562 & 58,5 & 48,2 & $-10,3$ & $0,004^{*}$ \\
\hline Consumo de verduras $\geq 5$ días/semana & 48,3 & 37,4 & $-10,9$ & $0,004^{*}$ & 37,6 & 29,4 & $-8,2$ & 0,668 \\
\hline Consumo de agua sin azúcar $\geq 5$ días/semana & 67,6 & 73,7 & 6,1 & $0,019^{*}$ & 69,1 & 58,5 & $-10,6$ & $0,002^{*}$ \\
\hline Consumo de galletas saladas $<2$ días/semana & 51,9 & 52,4 & 0,5 & 0,936 & 44,7 & 33,0 & $-11,7$ & $0,002^{*}$ \\
\hline Consumo de galletas dulces $<2$ días/semana & 65,2 & 62,8 & $-2,4$ & 0,453 & 56,0 & 43,3 & $-12,8$ & $0,002^{*}$ \\
\hline Consumo de jugos envasados $<2$ días/semana & 54,1 & 54,3 & 0,2 & 0,999 & 48,6 & 37,9 & $-10,6$ & $0,006^{*}$ \\
\hline Consumo de gaseosas $<2$ días/semana & 62,3 & 66,2 & 3,9 & 0,174 & 55,3 & 50,4 & $-5,0$ & 0,202 \\
\hline
\end{tabular}

† Prueba de Mc Nemar; ${ }^{*} p<0,05$

IE: institución educativa.

Aun cuando se encontraron cambios significativos en los conocimientos en nutrición, las conductas adecuadas en el consumo de alimentos y el nivel de actividad física en el grupo intervenido, no se encontró reducción en el puntaje $Z$ del IMC o el índice cintura/talla, lo cual es consistente con otros estudios que dan cuenta de efectos sobre variables biológicas en estudios de mediano y largo plazo ${ }^{(25,26) ;}$ en nuestro caso, la evaluación de impacto se realizó al primer año de implementar la intervención.

A pesar de que diversas revisiones sistemáticas muestran que las intervenciones basadas en escuelas para prevenir la enfermedad son efectivas ${ }^{(6-8)}$, en nuestro país solo encontramos un estudio controlado que evaluó la efectividad de este tipo de intervenciones. A diferencia de nuestros resultados, Gago et al. encontraron efectos al año de implementar una intervención multisectorial en educación alimentaria-nutricional en la prevención del sobrepeso u obesidad en escolares de IE de un distrito de Lima ${ }^{(27)}$. Sin embargo, este estudio solo abordó el componente alimentario y no reportó indicadores de proceso para evidenciar la calidad, la cobertura de la intervención ni la plausibilidad biológica que requiere una evaluación para atribuir efectos a la intervención ${ }^{(28)}$.

Respecto al impacto de "Como Jugando" sobre la frecuencia de consumo de alimentos, se encontraron resultados positivos en el consumo de frutas, agua sin azúcar, galletas (dulces y saladas) y jugos envasados; sin embargo, también se observó un efecto no deseado en el caso de verduras, pues en las IE de intervención hubo una reducción significativa de la conducta recomendada. Este resultado podría deberse a que el énfasis de "Como Jugando" se dio, sobre todo, para alimentos que se consumían en la IE y que formaban parte del refrigerio escolar que de forma regular no incluye verduras. Resultados en parte similares son reportados por un ensayo comunitario en Colombia que buscaba incrementar el consumo de frutas y verduras (mejoró el consumo de frutas, pero no el de verduras), los autores mencionaban que en el corto tiempo de intervención, las principales razones por las que no se logró el impacto ${ }^{(29)}$ fueron el no conocer los beneficios de las verduras o cómo prepararlas, así como no disponer de tiempo para cocinarlas.

Otros estudios en Latinoamérica han reportado resultados similares al nuestro respecto al efecto de intervenciones basadas en escuelas sobre las conductas adecuadas para elegir alimentos saludables y actividad física ${ }^{(12,15)}$, mientras que otros estudios refieren efectividad para mantener el IMC ${ }^{(13)} \mathrm{e}$, incluso, reducir el incremento del IMC en niños intervenidos. Por su parte, en Chile también hay estudios que reportan el efecto de intervenciones integrales sobre el IMC ${ }^{(10,11)}$.

Una de las limitaciones del estudio fue que la asignación del tratamiento no se pudo aleatorizar debido a que la intervención requería algunas condiciones mínimas de las IE de intervención como garantizar tiempo disponible para

Tabla 3. Impacto de la intervención educativa-motivacional "Como Jugando" sobre el conocimiento en nutrición y nivel de actividad física

\begin{tabular}{|c|c|c|c|c|c|c|c|c|}
\hline \multirow{2}{*}{$\begin{array}{l}\text { Indicadores } \\
\text { secundarios }\end{array}$} & \multicolumn{3}{|c|}{ Intervención } & \multicolumn{3}{|c|}{ Control } & \multirow{2}{*}{$\begin{array}{l}\text { OR crudo } \\
(95 \% \text { IC) }\end{array}$} & \multirow{2}{*}{$\begin{array}{l}\text { OR ajustado }{ }^{\dagger} \\
(95 \% \text { IC) }\end{array}$} \\
\hline & Antes & Después & $\begin{array}{c}\text { Cambio } \\
(\%)\end{array}$ & Antes & Después & $\begin{array}{c}\text { Cambio } \\
(\%)\end{array}$ & & \\
\hline $\begin{array}{l}\text { Escolares con } \\
\text { conocimiento } \\
\text { adecuado en nutrición }\end{array}$ & $\begin{array}{c}148 / 414 \\
(35,7)\end{array}$ & $\begin{array}{c}322 / 414 \\
(77,8)\end{array}$ & 42,1 & $\begin{array}{c}102 / 282 \\
(36,2)\end{array}$ & $\begin{array}{c}169 / 282 \\
(59,9)\end{array}$ & 23,7 & $\begin{array}{c}1,42 \\
(1,15 ; 1,76)^{*}\end{array}$ & $\begin{array}{c}1,46 \\
(1,17 ; 1,81)^{\star}\end{array}$ \\
\hline $\begin{array}{l}\text { Escolares con nivel de } \\
\text { actividad física mala }\end{array}$ & $\begin{array}{c}89 / 414 \\
(21,5)\end{array}$ & $\begin{array}{c}125 / 414 \\
(30,2)\end{array}$ & 8,7 & $\begin{array}{c}76 / 282 \\
(27,0)\end{array}$ & $\begin{array}{c}114 / 282 \\
(40,4)\end{array}$ & 13,4 & $\begin{array}{c}0,63 \\
(0,46 ; 0,87)^{*}\end{array}$ & $\begin{array}{c}0,63 \\
(0,46 ; 0,87)^{\star}\end{array}$ \\
\hline
\end{tabular}

†Ajustada por pobreza en el hogar ( $\geq 1$ necesidad básica insatisfecha), edad y sexo del escolar, edad, años de estudio y situación laboral de la madre; ${ }^{*} p<0,05$. 
Tabla 4. Impacto de la intervención educativa motivacional "Como Jugando" sobre el IMC y el Índice cintura/talla

\begin{tabular}{|c|c|c|c|c|c|c|c|c|}
\hline \multirow[b]{2}{*}{ Indicadores principales } & \multicolumn{3}{|c|}{ Intervención } & \multicolumn{3}{|c|}{ Control } & \multirow{2}{*}{$\begin{array}{l}\text { Diferencia de } \\
\text { medias } \\
\text { (IC 95\%) }\end{array}$} & \multirow{2}{*}{$\begin{array}{l}\text { Diferencia de } \\
\text { medias ajustada* } \\
\text { (IC 95\%) }\end{array}$} \\
\hline & $\begin{array}{l}\text { Antes } \\
(\bar{x})\end{array}$ & $\begin{array}{c}\text { Después } \\
(\bar{x})\end{array}$ & $\begin{array}{c}\text { Cambio } \\
(\bar{x})\end{array}$ & $\begin{array}{c}\text { Antes } \\
(\overline{\mathrm{x}})\end{array}$ & $\begin{array}{c}\text { Después } \\
(\bar{x})\end{array}$ & $\begin{array}{c}\text { Cambio } \\
(\bar{x})\end{array}$ & & \\
\hline Puntaje Z de IMC/edad & 1,19 & 1,24 & 0,05 & 0,08 & 0,96 & 0,08 & $-0,03(-0,11 ; 0,04)$ & $-0,04(-0,11 ; 0,03)$ \\
\hline Îndice Cintura/Talla & 0,51 & 0,50 & $-0,05$ & 0,49 & 0,49 & $-0,04$ & $-0,00(-0,00 ; 0,00)^{\dagger}$ & $-0,00(-0,00 ; 0,00)^{\dagger}$ \\
\hline
\end{tabular}

*Ajustada por pobreza en el hogar ( $\geq 1$ necesidad básica insatisfecha), edad y sexo del escolar, edad, años de estudio y situación laboral de la madre. + Valores inferiores a las centésimas

IMC: índice de masa corporal; $\bar{x}$ : media aritmética; IC 95\%: intervalo de confianza al 95\%

desarrollar las sesiones educativas, disposición de los docentes para ser capacitados y el involucramiento para destinar espacios para actividad física, así como asumir un rol más crítico para supervisar la oferta de alimentos dentro de la IE; bajo esta premisa, se seleccionaron 2 IE que se comprometieron a cumplir estos acuerdos. Otra limitación es que el estudio se desarrolló en 4 IE de un distrito de Lima, por lo que los resultados no se pueden extrapolar a otros ámbitos.

A nivel metodológico, una limitación potencial son los supuestos del análisis de diferencias en diferencias, que requiere garantizar una comportamiento constante de los indicadores previo a la estimación basal; en nuestro caso, no se tuvieron mediciones antes de la línea basal que nos permitieran asegurar tendencias iguales en ambos grupos. Sin embargo, las IE incluidas en el estudio tienen características similares en cuanto a la población escolar, ubicación e instalaciones por lo que esperamos que la dinámica de la evolución de peso en los niños de las escuelas sean similares; asimismo, el modelo de evaluación al aplicar las dobles diferencias "elimina" las diferencias iniciales y los resultados se ajustaron por variables observables para mejorar la estimación. Por otra parte, en cuanto a métodos, el consumo de alimentos, se recogió con una frecuencia cualitativa de consumo, por lo que el cambio se midió en patrones de alimentación y no en forma cuantitativa, al respecto, otros estudios han aplicado este método de consumo de alimentos para evaluar la efectividad de la intervención ${ }^{(13,28)}$. Asimismo, el nivel de actividad física se hizo con cuestionario, lo cual torna subjetiva, en parte, la determinación, al respecto, cabe precisar que este instrumento ha sido validado con acelerómetros triaxiales para determinar su utilidad, varios estudios han evaluado el efecto de una intervención con cuestionarios de actividad física, considerando que la actividad física en escolares tiene un patrón regular ${ }^{(11,13,30)}$.

En conclusión, el estudio muestra que al primer año de intervención "Como Jugando" no tuvo impacto sobre el IMC; sin embargo, tiene efecto atribuible sobre el aumento de conocimientos en nutrición, reduce la probabilidad de tener un nivel malo de actividad física y mantiene o aumenta la frecuencia adecuada del consumo de frutas, agua sin azúcar y mantiene un consumo menor a dos veces por semana de jugos envasados y galletas dulces $y$ saladas. Es necesario continuar evaluando la intervención "Como Jugando" para estimar el impacto sobre indicadores biológicos que cambian de mediano a largo plazo.

Contribuciones de autoría: JPA y JP han participado en la concepción y diseño del artículo. JPA, JP y WB participaron en la recolección, análisis e interpretación de los datos. Todos los autores participaron en la redacción del borrador dl artículo, revisaron críticamente el artículo y aprobaron la versión final.

Fuentes de financiamiento: el proyecto fue financiado por el Instituto Nacional de Salud.

Declaración de conflictos de interés: los autores declaran no tener conflictos de interés.

\section{REFERENCIAS BIBLIOGRÁFICAS}

1. Roberto CA, Swinburn B, Hawkes C, Huang TT, Costa SA, Ashe M, et al. Patchy progress on obesity prevention: emerging examples, entrenched barriers, and new thinking. Lancet. 2015;385(9985):2400-9. doi: 10.1016/ S0140-6736(14)61744-X

2. Lobstein T, Jackson-Leach R, Moodie ML, Hall KD, Gortmaker SL, Swinburn BA, et al. Child and adolescent obesity: part of a bigger picture. Lancet. 2015;385(9986):2510-20. doi: 10.1016/ S0140-6736(14)61746-3
3. $\mathrm{Ng} \mathrm{M}$, Fleming $\mathrm{T}$, Robinson $\mathrm{M}$, Thomson B, Graetz N, Margono C, et al. Global, regional, and national prevalence of overweight and obesity in children and adults during 1980-2013: a systematic analysis for the Global Burden of Disease Study 2013. Lancet; 384(9945):766-81. doi: 10.1016/S0140-6736 (14)60460-8.

4. Aparco JP, Bautista-Olórtegui W, AsteteRobilliard L, Pillaca J. Evaluación del estado nutricional, patrones de consumo alimentario y de actividad física en escolares del Cercado de Lima. Rev Peru
Med Exp Salud Publica. 2016;33(4):6339. doi: 10.17843/rpmesp.2016.334.2545

5. Gearhardt AN, Bragg MA, Pearl RL, Schvey NA, Roberto CA, Brownell KD. Obesity and public policy. Annu Rev Clin Psychol. 2012;8:405-30. doi: 10.1146/ annurev-clinpsy-032511-143129

6. Verrotti A, Penta L, Zenzeri L, Agostinelli $S$, De Feo P. Childhood obesity: prevention and strategies of intervention. A systematic review of school-based interventions in primary schools. J Endocrinol Invest. 2014;37(12):1155-64. doi: 10.1007/s40618-014-0153-y. 
7. Brown EC, Buchan DS, Baker JS, Wyatt FB, Bocalini DS, Kilgore L. A systematised review of primary school whole class child obesity interventions: effectiveness, characteristics, and strategies. Biomed Res Int. 2016;2016:4902714. doi: $10.1155 / 2016 / 4902714$

8. Amini M, Djazayery A, Majdzadeh R, Taghdisi MH, Jazayeri S. Effect of schoolbased interventions to control childhood obesity: a review of reviews. Int J Prev Med. 2015;6:68. doi: 10.4103/20087802.162059 .

9. Guerra PH, da Silveira JA, Salvador EP. Physical activity and nutrition education at the school environment aimed at preventing childhood obesity: evidence from systematic reviews. J Pediatr (Rio J). 2016;92(1):15-23. doi: 10.1016/j.jped.2015.06.005

10. Kain J, Leyton B, Concha F, Weisstaub SG, Lobos L, Bustos N, et al. Evaluación de una intervención en educación alimentaria y actividad física para prevenir obesidad infantil en escuelas públicas de Santiago de Chile. ALAN. 2012;62(1):60-7.

11. Ratner R, Durán $S$, Garrido $M J$, Balmaceda $S$, Jadue L, Atalah E. Impacto de una intervención en alimentación y actividad física sobre la prevalencia de obesidad en escolares. Nutr Hosp. 2013;28(5):1508-14. doi: 10.3305/ nh.2013.28.5.6644

12. Vio F, Salinas J, Montenegro E, González CG, Lera L. Efecto de una intervención educativa en alimentación saludable en profesores, y niños prescolares y escolares de la región de Valparaíso, Chile. Nutr Hosp. 2014; 29(6):1298-304. doi: 10.3305/nh.2014.29.6.7409

13. Shamah T, Morales C, Amaya C, Salazar A, Jiménez A, Méndez I. Effectiveness of a diet and physical activity promotion strategy on the prevention of obesity in Mexican school children. BMC Public Health. 2012;12:152. doi: 10.1186/1471-2458-12-152.

14. Alvirde-Garcia Alvirde-García U, Rodríguez-Guerrero AJ, Henao-Morán S, Gómez-Pérez FJ, Aguilar-Salinas CA. Resultados de un programa comunitario de intervención en el estilo de vida en niños. Salud Publica Mex. 2013;55(Supp 3):S406-14.

15. Safdie M, Jennings-Aburto N, Lévesque L, Janssen I, Campirano-Núñez F, López-Olmedo N, Aburto T, Rivera JA. Impact of a school-based intervention program on obesity risk factors in Mexican children. Salud Publica Mex. 2013;55(Suppl 3):374-87.

16. Bandura A. Social foundations of thought and action. Englewood Cliffs, NJ: Prentice Hall; 1986.

17. Contento IR. Nutrition education: linking research, theory and practice. 3a ed. Sudbury, MA: Jones and Bartlett Publishers; 2015.

18. Hawkes C, Smith TG, Jewell J, Wardle J, Hammond RA, Friel S, et al. Smart food policies for obesity prevention. Lancet. 2015;385(9985):2410-21. doi: 10.1016/ S0140-6736(14)61745-1

19. Shadish WR, Cook TD, Campbell DT. Experimental and quasi-experimental designs for generalized causal inference. New York: Houghton Mifflin Company; 2002.

20. Instituto Nacional de Estadística e Informática (INEI). Encuesta Nacional de Hogares 2010, Condiciones de vida y Pobreza, 2do trimestre [Internet]. Lima, Perú: INEI; 2011 [citado 13 de noviembre de 2013].

21. Muñoz-Cano J, Pérez-Sánchez $S$, Córdova-Hernández J, Boldo-León X. El índice cintura/talla como indicador de riesgo para enfermedades crónicas en una muestra de escolares. Salud en Tabasco. 2010;16(2-3):921-7.

22. Serra Majem L., Aranceta J, Mataix J. Documento de consenso. Guías alimentarias para la población española. SENC. Barcelona: SG editores; 1995. p. 301-8.

23. Godard MC, Rodríguez MdP, Díaz N, Lera L, Salazar G, Burrows R. Valor de un test clínico para evaluar actividad física en niños. Rev. Méd. Chile. 2008;136(9):1155-62.

24. Wooldridge JM. Introductory Econometrics: A Modern Approach. 5a ed. Cincinnati, OH: South-Western College Publishing; 2013
25. Wang Y, Cai L, Wu Y, Wilson RF, Weston $\mathrm{C}$, Fawole $\mathrm{O}$, et al. What childhood obesity prevention programmes work? A systematic review and meta-analysis. Obes Rev. 2015;16(7):547-65. doi: 10.1111/obr.12277

26. Kain J, Concha F, Moreno L, Leyton B. School-based obesity prevention intervention in Chilean children: effective in controlling, but not reducing obesity. J Obes. 2014;2014:618293. doi: $10.1155 / 2014 / 618293$

27. Gago J, Rosas O, Huayna M, Jiménez D, Córdova F, Navarro A, et al. Efectividad de una intervención multisectorial en educación alimentaria nutricional para prevenir y controlar el sobrepeso y la obesidad en escolares de cuatro instituciones educativas públicas del distrito de Villa El Salvador. Rev Peru Epidemiol. 2014;18(3):e04.

28. Habicht JP, Victora CG, Vaughan JP. Evaluation designs for adequacy, plausibility and probability of public health programme performance and impact. Int J Epidemiol. 1999;28(1):10-8.

29. Prada GE, Dubeibe-Blanco LY, Herrán OF, Herrera-Anaya M. Evaluación del impacto de un ensayo comunitario sobre el consumo de frutas y verduras en Colombia. Salud Publica Mex. 2007;49(1):11-9. 10.1590/S003636342007000100003

30. Llargues E, Franco R, Recasens A, Nadal A, Vila M, Pérez MJ, et al. Assessment of a school-based intervention in eating habits and physical activity in school children: the AVall study. J Epidemiol Community Health. 2011; 65(10):896-901. doi: 10.1136/jech.2009.102319.

Correspondencia: Juan Pablo Aparco Balboa Dirección: Jr. Tizón y Bueno 276- Jesús María. Lima, Perú.

Teléfono: (+51) 7480000 anexo 6626

Correoelectrónico:japarco@ins.gob.pe 